Research Paper

\title{
Longitudinal assessment of symptoms and risk of SARS-CoV-2 infection in healthcare workers across 5 hospitals to understand ethnic differences in infection risk.
}

\author{
Ana M Valdes, Professor ${ }^{1, *, a}$, James C Moon, Professor ${ }^{2,3, b}$, Amrita Vijay, Research Fellow ${ }^{1, c}$, \\ Nish Chaturvedi, Professor ${ }^{4, \mathrm{~d}}$, Alan Norrish, Senior Research Fellow ${ }^{1, \mathrm{e}}$, \\ Adeel Ikram, Registrar ${ }^{1, \mathrm{f}}$, Simon Craxford, Research Fellow ${ }^{1, \mathrm{~g}}$, Lola M.L. Cusin, PhD Student ${ }^{5, \mathrm{~h}}$, \\ Jessica Nightingale, Clinical Research Manager ${ }^{1, i}$, Amanda Semper, Principal scientist ${ }^{6, j}$, \\ Timothy Brooks, Clinical Services Director ${ }^{6, k}$, Aine McKnight, Professor ${ }^{7,1}$, \\ Hibba Kurdi, Research Fellow ${ }^{2, m}$, Cristina Menni, Lecturer ${ }^{8, n}$, \\ Patrick Tighe, Associate Professor ${ }^{5,0}$, Mahdad Noursadeghi, Professor ${ }^{9, p}$, \\ Guruprasad Aithal, Professor ${ }^{10, \mathrm{q}}$, Thomas A Treibel, Associate Professor ${ }^{2,3, \mathrm{r}}$, \\ Benjamin J Ollivere, Professor ${ }^{1, \mathrm{~s}}$, Charlotte Manisty, Associate Professor ${ }^{2,3, \mathrm{t}}$ \\ ${ }^{1}$ Division of Rheumatology, Orthopaedics and Dermatology, School of Medicine, University of Nottingham, Nottingham, NG5 1PB, UK \\ ${ }^{2}$ Barts Heart Centre, St. Bartholomew's Hospital, London, United Kingdom \\ ${ }^{3}$ Institute of Cardiovascular Sciences, University College London, London, United Kingdom. \\ ${ }^{4}$ MRC Unit for Lifelong Health and Ageing at UCL, University College London, London, United Kingdom \\ ${ }^{5}$ School of Life Sciences, University of Nottingham, NG7 2RB \\ ${ }^{6}$ National Infection Service, Public Health England, Porton Down, UK \\ ${ }^{7}$ Blizard Institute, Barts and the London School of Medicine and Dentistry, Queen Mary University of London, London, UK \\ ${ }^{8}$ Department of Twin Research and Genetic Epidemiology, King's College London, London SE1 7EH, UK \\ ${ }^{9}$ Division of Infection and Immunity, University College London, London, UK \\ ${ }^{10}$ Nottingham Digestive Disease Centre, University of Nottingham School of Medicine, Nottingham, UK
}

\footnotetext{
Funding: PANTHER study funded from the UKRI/MRC (Cov-0331 - MR/V027883/1), with additional institutional support from the Nottingham NIHR BRC. Funding for COVIDsor-

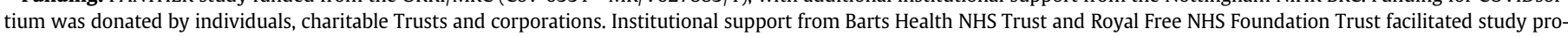
cesses, in partnership with University College London and Queen Mary University London.

* Corresponding Author: Professor Ana Valdes, University of Nottingham School of Medicine, Nottingham, United Kingdom, Tel: (+44)0115 823 1954

E-mail address: ana.valdes@nottingham.ac.uk (A.M. Valdes).

a $0000-0003-1141-4471$

b $0000-0001-8071-1491$

c $0000-0002-9595-5680$

d $0000-0002-4774-0853$

e $0000-0003-3735-1042$

f $0000-0002-7520-6949$

g $0000-0002-4672-4587$

h $0000-0002-1437-8034$

i $0000-0001-8359-5705$

j $0000-0003-3656-1363$

k 0000-0002-3783-1284

${ }^{1} 0000-0002-8113-817 \mathrm{X}$

m 0000-0002-4807-0636

n 0000-0001-9790-0571

o 0000-0001-8326-093X

p 0000-0002-4774-0853

q $0000-0003-3924-4830$

r $0000-0003-1560-7414$

s $0000-0002-1410-1756$

t $0000-0003-0245-7090$
} 


\section{A R T I C L E I N F O}

\section{Article History:}

Received 8 January 2021

Revised 23 March 2021

Accepted 24 March 2021

Available online xxx

\section{Keywords:}

Covid-19

seropositivity

Healthcare workers

ethnicity
A B S T R A C T

Background: : Healthcare workers (HCWs) have increased rates of SARS-CoV-2 infection compared with the general population. We aimed to understand ethnic differences in SARS-CoV-2 seropositivity among hospital healthcare workers depending on their hospital role, socioeconomic status, Covid-19 symptoms and basic demographics.

Methods: A prospective longitudinal observational cohort study. 1364 HCWs at five UK hospitals were studied with up to 16 weeks of symptom questionnaires and antibody testing (to both nucleocapsid and spike protein) during the first UK wave in five NHS hospitals between March 20 and July 10 2020. The main outcome measures were SARS-CoV-2 infection (seropositivity at any time-point) and symptoms. Registration number: NCT04318314.

Findings: 272 of 1364 HCWs (mean age 40.7 years, 72\% female, 74\% White, $\geq 6$ samples per participant) seroconverted, reporting predominantly mild or no symptoms. Seropositivity was lower in Intensive Therapy Unit (ITU) workers (OR=0.44 95\%CI 0.24, 0.77; $\mathrm{p}=0.0035$ ). Seropositivity was higher in Black (compared to White) participants, independent of age, sex, role and index of multiple deprivation (OR=2.61 95\%CI 1.47 $4.62 \mathrm{p}=0.0009$ ). No association was seen between White HCWs and other minority ethnic groups.

Interpretation: In the UK first wave, Black ethnicity (but not other ethnicities) more than doubled HCWs likelihood of seropositivity, independent of age, sex, measured socio-economic factors and hospital role.

(C) 2021 Published by Elsevier Ltd. This is an open access article under the CC BY-NC-ND license

(http://creativecommons.org/licenses/by-nc-nd/4.0/)

\section{Research in context}

\section{Evidence before this study}

We searched PubMed for articles published between Jan 1 and December 18, 2020, with the terms "Covid-19", "healthcare workers", and "ethnicity". We did not restrict our search by language or type of publication. Patients from many Black and minority ethnic (BAME) groups have been shown to have higher rates of hospitalisation and worse outcomes with SARS-CoV-2 infection, with differences in comorbidities and socio-economic factors considered important causes. Healthcare workers (HCWs) have increased rates of SARS-CoV-2 infection compared with the general population due at least in part to high levels of occupational exposure. Understanding true differences in infection rates between ethnic groups also requires data from surveillance screening across asymptomatic populations and adjustment for socio-economic and exposure levels. Our PubMed search yielded no investigations carrying out separate analyses of the various ethnicities that are categorised as BAME in the UK.

\section{Added value of this study}

We carried out a longitudinal serological study among 1364 healthcare workers in 5 hospitals in England adjusting for surrogates of socioeconomic status, hospital role, access to PPE, age and sex and presence of Covid-19 symptoms and analysed the role of the various ethnicities categorised as BAME in the UK with regards to overall seropositivity.

\section{Implications of all available evidence}

Our study suggests that HCWs of Black ethnicity (but not other minority ethnic groups) have higher overall rates of SARS-CoV2 seropositivity than their White counterparts, even after adjustment for demographics, socio-economic status, presence of Covid-19 symptoms and exposure, suggesting a higher ability to mount an antibody response to the virus. HCWs working on ITU had lower rates of SARS-CoV-2 infection during the first wave of the COVID-19 pandemic compared with other frontline HCWs.

\section{Introduction}

Ethnic differences in severe disease hospitalisation [1] and mortality [2] emerged early in the SARS-CoV-2 pandemic. Proposed explanations include the impact of comorbidities, genetic and socio-economic factors, including access to healthcare, personal protective (PPE) equipment, behavioural and occupational influences [3]. However, most SARS-CoV-2 infections are non-hospitalised with approximately onethird asymptomatic [4]. Large-scale community cross-sectional epidemiological studies have reported higher infection prevalence in certain ethnic minority populations [5]. These studies however typically report PCR tests in symptomatic individuals or single time point community surveillance [6], underestimating true case ascertainment. Even for population serology studies at scale, limitations remain including recall bias (for symptoms), reduced test sensitivity or specificity (PCR/antigen testing especially at point-of-care), variable/inappropriate timing of tests (prior to sero-conversion; after sero-reversion) and challenges with adjusting for exposure and socio-economic status.

Frontline healthcare workers (HCWs) are at higher SARS-CoV-2 infection risk $[7,8,9]$ with reported estimates from 3.4 to 18 times higher than the general population [8-10]. Understanding HCW infection is important. Nosocomial transmission can make hospitals pandemic amplifiers/sustainers [11] and lessons from the first wave are swiftly needed as subsequent waves emerge. HCWs are also work-force representative, easier to study (e.g. during lock-down), have good ethnic minority representation [5,12] and have many societal socio-economic and behavioural confounders removed or measurable (by NHS role), making them useful to explore ethnic differences in SARS-CoV-2 transmission dynamics. Finally, working in an environment where there is substantial risk of sustained exposure, HCW cohort studies allow an assessment of the impact of mitigation strategies such as PPE [13].

Using best available technology to define SARS-CoV-2 infection and symptoms (longitudinal data collection, multi-timepoint accurate lab-based antibody assays for two antigens), this study assessed differences in symptomatology, serological prevalence and infection severity in HCWs by ethnicity across five hospitals, correcting for demographic and socio-economic differences where feasible.

\section{Methods}

\section{Healthcare worker cohorts}

A five hospital HCW longitudinal study $(n=1,364)$ of UK first wave SARS-CoV-2 infection consisting of two initially independent studies 
(PANTHER, Nottingham: Nottingham City Hospital and Queen's Medical Centre - both Nottingham University Hospital NHS trust; COVIDsortium, London: St Bartholomew's, Nightingale and Royal Free Hospitals) that were methodologically aligned for scale in April 2020 (NCT04318314). London ethical approval was South Central - Oxford A Research Ethics Committee, reference 20/SC/0149. Nottingham was initially under a Human Tissue Authority licence in Nottingham (Licence number: 11035) and subsequently also North West - Greater Manchester South Research Ethics Committee, reference 20/NW/ 0395. All participants provided written informed consent prior to completing the questionnaire and providing blood samples.

Both studies recruited asymptomatic HCWs attending work. Nurses, doctors, allied health professionals and other frontline health-care workers were recruited in Nottingham and London, with additional staff from other workforce groups (hospital administrative staff, porters and cleaners) in London. Both studies started early in their local pandemic waves ( $23^{\text {rd }}$ March 2020, London - day one of first UK lockdown20 April in Nottingham) Results presented here.

Data included in this study refers to weekly or fortnightly serological and symptoms data up to 10 July 2020. Data was stored in MS Access databases, C Manisty, J Moon A Valdes had access to the data collected in London, J Nightingale, B Ollivere, A Valdes had access to the data collected in Nottingham with sample collection and detailed questionnaires (demographics including self-declared ethnicity split as white of any origin (British white, Irish white, or white of any other origin), Black [both African or Caribbean], South Asian, East Asian, mixed, other), occupational roles and ward type, comorbidities, travel, exposure, PPE, symptoms). Participants attended for follow-up if asymptomatic and working; those with symptoms or in self-isolation resumed on return to work, supplying prior symptoms with minimal delay to reduce recall bias. Further study details are published elsewhere [14,15]. Blood serum samples for serological assessment were immediately spun and stored at $-80^{\circ} \mathrm{C}$. Both studies collected information on hospital role, age, gender and body mass index.

\section{Anti-S1 and anti-nucleocapsid assays}

Both studies performed serial SARS-CoV-2 serology testing assessing antibodies to both spike (S1) and nucleocapsid protein (NP).

The London samples were analysed using validated commercial assays; the Euroimmun anti-SARS-CoV-2 enzyme-linked immunosorbent assay (ELISA) targeting IgG specific for S1 [16], and the Roche Elecsys Anti-SARS-CoV-2 electrochemiluminescence immunoassay (ECLIA) that detects antibodies (including $\operatorname{IgG}$ ) for $\mathrm{N}$ protein [17]. These were undertaken at the Rare \& Imported Pathogens Laboratory at Public Health England using standard protocols, total 12,990 tests. Positive was defined as (Euroimmun) a ratio of sample OD to calibrator OD $>1.1$, and (Roche) a electrochemiluminescence sample to lotspecific cutoff index $>1$, as per manufacturers' instructions. Reported assay sensitivity (92.3\% and $96.2 \%-100 \%$ for Roche and Euroimmune respectively) and specificities (100\%) are high [18,19].

The Nottingham study used in-house ELISA robotically delivered assays cross-validated by the same Public Health England laboratory (Porton-Down, UK). Assay details are in supplementary methods. In brief, they were ELISAs to $\mathrm{S} 1$ and $\mathrm{N}$ protein detecting immunoglobulin G. Individuals were classified as seropositive if they had a positive titre to either protein at any time point. Seropositivity was defined as samples where the average measurement of the duplicates exceeded two-times the median value for the pooled negative controls.

\section{Symptoms}

Individuals were classified as having case-definition symptoms, if at any time point they reported characteristic symptoms (fever, dry cough, loss of sense of smell or taste) using the symptoms-based model developed previously [20], or if they had to self-isolate due to symptoms of COVID-19. Individuals were classified as asymptomatic if they either did not report any symptoms or reported symptoms not matching the case definition (i.e. the clinical criteria for assessing possibility of infection with COVID-19 based on characteristic symptoms).

\section{Statistical analysis}

Associations between seropositivity and demographic, social or hospital roles were assessed by standard logistic regression, adjusting for all available covariates. Missing or unspecified data was imputed to the mean value. Where missing data was more than $10 \%$ sub-analyses were run only on individuals who had the trait with and without adjustment Because of potential differences in serological assays between London and Nottingham, results were not pooled. Rather, fixed effects meta-analyses were performed on odds ratios and standard error estimates derived from the logistic regressions. The heterogeneity variance was computed for each meta analysis and where it was $\tau^{2}>0.10$ a DerSimonian Laird random effects estimate was used. All analyses were carried out using R version 3.6.1 (2019-0705) (The R Foundation for Statistical Computing).

\section{Socio-economic confounders adjustment}

We assigned each participant based on their postcode, the Index of Multiple Deprivation (IMD) decile for their geographical area. The English IMD, the official measure of relative deprivation in England, follows an established methodological framework in broadly defining deprivation to encompass a wide range of an individual's living conditions [21]. This area-based measure of deprivation is derived from ranking the 32,844 Lower Layer Super Output Areas (LSOAs, the geographic unit used by the Office of National statistics) in England and dividing them into deciles.

\section{Role of funding source}

The funding bodies had no role in study design, data collection, or analysis of the data.

\section{Results}

The descriptive characteristics of both HCW cohorts are presented in Table 1 and individual cohorts have been previously described. ${ }^{14,15}$ In short, mean age across the combined cohorts was 40.7 years, SD,11.6, range $18-69$, and $71.6 \%$ female. The ethnic mix of the cohort was $73.5 \%$ of White, $4.3 \%$ Black ethnicity, $9.4 \%$ South Asian, $5.7 \%$ East Asian, $6.2 \%$ other or mixed. This is a similar proportion of ethnic minorities as in the NHS at large (26.3 vs $22.1 \%$ in the NHS [12] and a slightly lower proportion of women (71.6\%) compared with $77 \%$ in the NHS at large [22].

The average number of antibody tests was 10 paired samples for each London HCW and 6.5 for each Nottingham HCW. 21.3\% of London HCWs (157 out of 731) and 18.2\% of Nottingham HCWs (115 out of 633) were seropositive denoting infection, with no significant difference between both cities overall $(\mathrm{P}=0.071)$. Infections were predominantly mild, with two participants (one of White ethnicity, one of East Asian) being hospitalised briefly, but with no requirement for ventilation and no deaths. Of the 272 participants seropositive at any time point, $24.8 \%$ participants reported case-definition symptoms at the time of or in the two months preceding seroconversion (i.e. seropositivity), and the remainder reported either atypical symptoms or were entirely asymptomatic. Typical symptoms were strongly associated with seropositivity $(\mathrm{OR}=3.66(2.73,4.90) \mathrm{p}=1 \times 10[-18])$; Figure 1). 
Table 1

Rates of SARS-CoV-2 seropositivity (IgGs against either nucleocapsid or spike 1 proteins) among healthcare workers from five hospitals. Individuals were assessed over a 13 (London) or 10 (Nottingham) week period between 23 March 2020 and 10 July 2020 and are considered seropositive if they showed seropositivity at any time point during this longitudinal assessment.

\begin{tabular}{|c|c|c|c|c|c|c|c|}
\hline & & \multicolumn{2}{|c|}{ London } & \multirow[b]{2}{*}{ Seropositive\% } & \multicolumn{2}{|c|}{ Nottingham } & \multirow[b]{2}{*}{ Seropositive\% } \\
\hline & & $\mathrm{Ab}-$ & $\mathrm{Ab}+$ & & $\mathrm{Ab}-$ & $\mathrm{Ab}+$ & \\
\hline \multirow[t]{5}{*}{ Hospital } & City Hospital & & & & 165 & 37 & $18.3 \%$ \\
\hline & $Q M C$ & & & & 353 & 78 & $17.9 \%$ \\
\hline & Barts/Nightingale & 403 & 101 & $20.0 \%$ & & & \\
\hline & Royal Free & 171 & 56 & $24.7 \%$ & & & \\
\hline & Total & 574 & 157 & $21.5 \%$ & 518 & 115 & $18.2 \%$ \\
\hline \multirow[t]{2}{*}{ Age } & Mean years & 37.8 & 39.4 & & 43.1 & 43.9 & \\
\hline & $(S D)$ & 10.9 & 11 & & 11.6 & 11.6 & \\
\hline \multirow[t]{2}{*}{ BMI } & Mean $\mathrm{kg} / \mathrm{m} 2$ & 25.0 & 25.6 & & 26.5 & 26.9 & \\
\hline & $(S D)$ & 4.5 & 4.4 & & 4.8 & 5.0 & \\
\hline \multirow[t]{3}{*}{ Sex } & M & 188 & 54 & $22.3 \%$ & 115 & 22 & $16.1 \%$ \\
\hline & $\mathrm{F}$ & 384 & 102 & $21.0 \%$ & 401 & 93 & $18.7 \%$ \\
\hline & Other/Unspec & 2 & 1 & $33.3 \%$ & 2 & 0 & $0.0 \%$ \\
\hline Case definition* & Yes & 111 & 71 & $39.0 \%$ & 108 & 42 & $28.0 \%$ \\
\hline $\begin{array}{l}\text { Covid-19 } \\
\text { symptoms }\end{array}$ & No & 463 & 86 & $15.7 \%$ & 410 & 73 & $15.1 \%$ \\
\hline \multirow[t]{8}{*}{ Ethnicity } & Asian & 126 & 25 & $16.6 \%$ & 46 & 12 & $20.7 \%$ \\
\hline & South Asian & 67 & 12 & $15.2 \%$ & 40 & 10 & $20.0 \%$ \\
\hline & East Asian & 57 & 13 & $18.6 \%$ & 6 & 2 & $25.0 \%$ \\
\hline & OtherAsian & 1 & 2 & & & & \\
\hline & Black & 24 & 18 & $42.9 \%$ & 10 & 7 & $41.2 \%$ \\
\hline & Other/Mixed & 46 & 6 & $11.5 \%$ & 24 & 9 & $27.3 \%$ \\
\hline & Unspecified & 6 & 1 & $14.3 \%$ & 1 & 0 & \\
\hline & White & 372 & 107 & $22.3 \%$ & 439 & 87 & $16.5 \%$ \\
\hline \multirow[t]{4}{*}{ IMD decile } & $1-3$ & 138 & 46 & $25.0 \%$ & 98 & 29 & $22.8 \%$ \\
\hline & $4-7$ & 265 & 76 & $22.3 \%$ & 177 & 38 & $17.7 \%$ \\
\hline & $8-10$ & 73 & 21 & $22.3 \%$ & 130 & 29 & $18.2 \%$ \\
\hline & Unspecified & 98 & 14 & $12.5 \%$ & 113 & 19 & $14.4 \%$ \\
\hline \multirow[t]{3}{*}{ Use of PPE } & ITU role & 109 & 17 & $13.5 \%$ & 36 & 1 & $2.7 \%$ \\
\hline & use PPE not ITU & 353 & 112 & $24.1 \%$ & 337 & 84 & $20.0 \%$ \\
\hline & other roles & 112 & 28 & $20.0 \%$ & 147 & 30 & $16.9 \%$ \\
\hline \multirow[t]{4}{*}{ Hospital role } & Doctor & 115 & 35 & $23.3 \%$ & 68 & 20 & $22.7 \%$ \\
\hline & Nurse/AHP & 386 & 98 & $20.2 \%$ & 312 & 67 & $17.7 \%$ \\
\hline & Clinical Support worker & 7 & 3 & $30.0 \%$ & 10 & 1 & $9.1 \%$ \\
\hline & other & 67 & 20 & $23.0 \%$ & 128 & 27 & $17.4 \%$ \\
\hline
\end{tabular}

ITU= intensive therapy unit; AHP = allied healthcare professional; clinical support worker includes portering and cleaning staff

* the clinical criteria for assessing possibility of infection with COVID-19 based on characteristic symptoms

Rates of infection were higher in HCWs from Black ethnic groups (42\%) than of White HCWs (19\%) (adj OR=2.61 (1.47, 4.62) p=0.0009), however there were no significant associations between seropositivity and ethnicity when South Asian, East Asian or mixed/other nonBlack minority ethnic groups were compared to White HCWs (Figure 1). Although we saw higher seropositivity among East Asian HCW in Nottingham, this result is derived from a very small sample size $(n=8)$ and is not observed in the larger London cohort which includes $n=70$ East Asian HCWs. No significant association was seen with regards to other/mixed ethnicity $(\mathrm{OR}=0.8195 \% \mathrm{CI} 0.42,1.57$ n.s.).

There were no associations of seropositivity with age, BMI (OR= $1.01 ; 95 \%$ CI 0.98-1.05), sex, IMD and use of PPE outside of an intensive therapy unit (ITU), (Figure 1). However, HCWs who had an ITU role had half the likelihood of being seropositive (OR=0.44 95\% 0.24 , $0.77 ; \mathrm{p}=0.0035$ ). No other significant differences in infection rates were found between different hospital roles (doctors compared with nurses and other allied healthcare professionals, or clinical support and administrative staff; Table 1).

Even after adjusting for other confounders (demographics, occupation and IMD), the association of increased risk of infection with Black ethnicity remained significant ( $\mathrm{OR}=2.61 \quad 95 \% \mathrm{CI} \quad 1.47-4.62$ $\mathrm{p}=0.0009$ ) (Figure 1). Across seropositive HCWs, there were no differences in timing of infection (with potential for recall bias) by ethnicity, or in antibody responses to infection, with similar durations of seropositivity, and peak antibody responses between different ethnic groups. The association between Black ethnicity and symptomatic infection (OR=2.57 95\%CI 1.20-5.09 p=0.0167) and between Black ethnicity and asymptomatic infection (OR=3.20 95\%CI $1.64-6.22$ $\mathrm{p}=0.0006$ ) in the two HCW cohorts are similar therefore we find no evidence for this association between Black ethnicity and seropositivity being in any way linked to the presence or absence of Covid-19 symptoms.

Finally, because IMD was missing for $17.8 \%$ of individuals we carried out sensitivity analyses only on participants who had complete data. The significant associations of seropositivity with Black ethnicity (OR= 2.61 95\% CI 1.47, 4.62), ITU role (OR= $0.4395 \% \mathrm{CI} 0.24,0.76)$ and Covid-19 symptoms (random effects OR=3.39 95\% CI 1.36,8.46) were extremely similar to those including imputed IMD data.

\section{Discussion}

These data show that, after adjusting for demographic and socioeconomic confounders and levels of exposure at work, Black HCWs 


\section{Ethnicity}
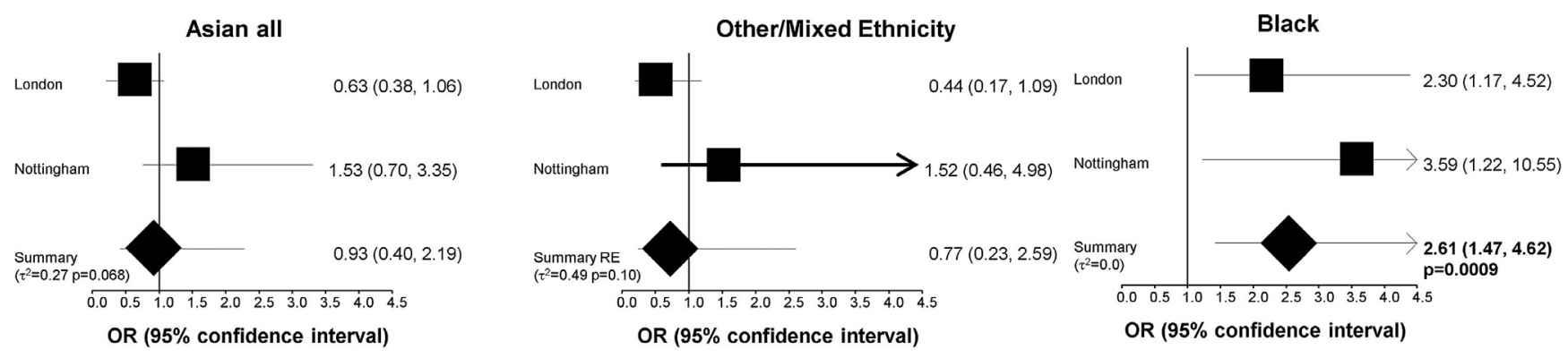

East Asian

South Asian
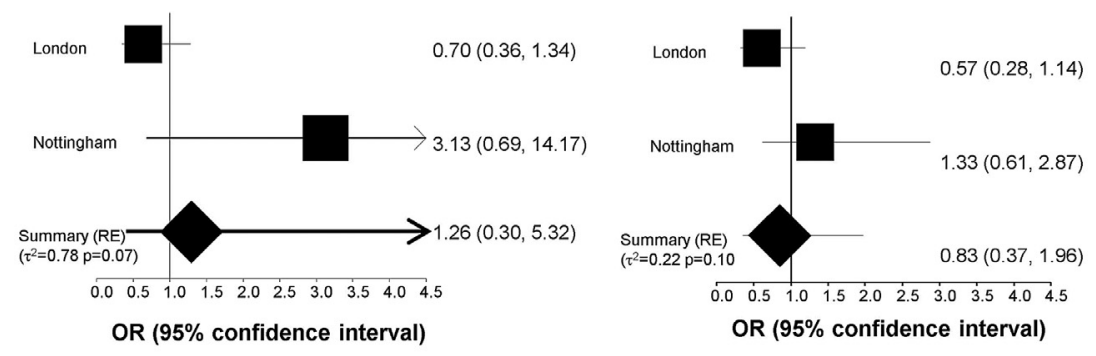

\section{Other demographics}
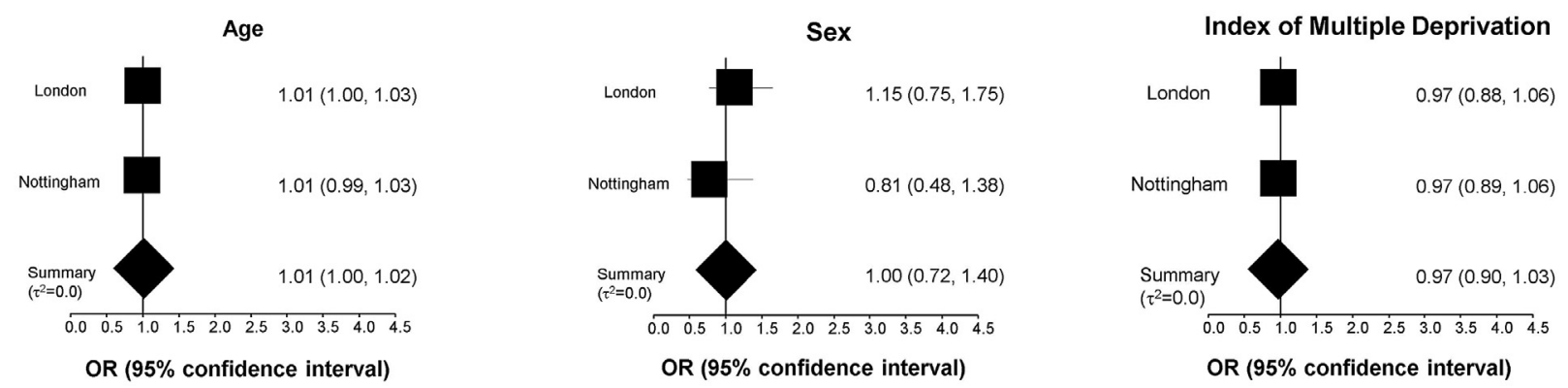

\section{Hospital Role}

\section{Covid-19 Symptoms}
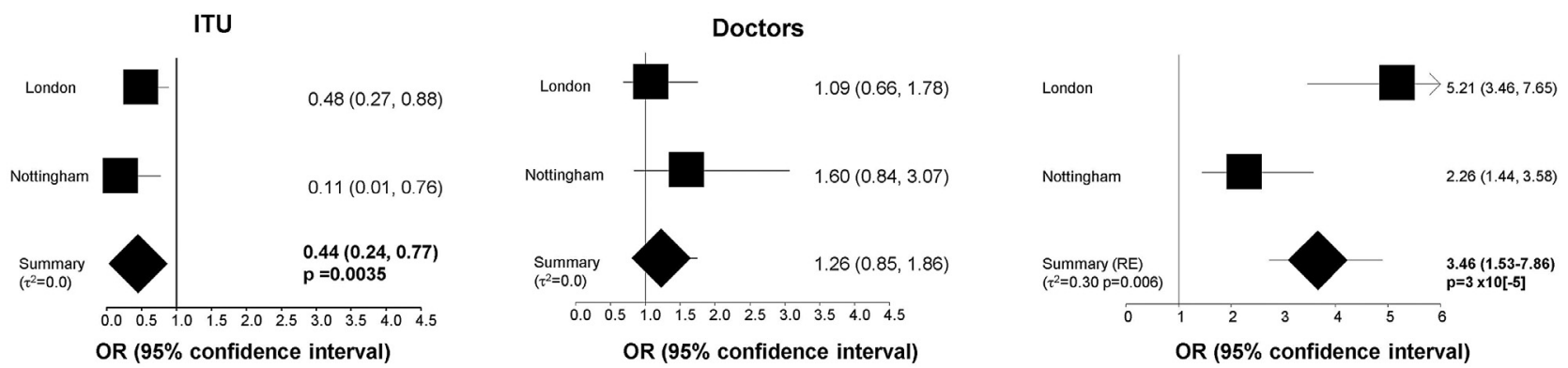

Figure 1. Association between SARS-COV-2 seropositivity over a 10-13 week period and demographic, hospital role and ethnicity in 1365 healthcare workers from London and Nottingham. All analyses are adjusted for age (per year), sex (male vs female), body mass index, index of multiple deprivation (IMD, per decile), hospital role (ITU vs non ITU, doctors vs other roles), presence of symptoms and ethnicity (vs Whites). Where the heterogeneity variance $(\tau 2)$ is equal to 0 the fixed effect meta-analysis estimates are the same as random effects. Where $\tau 2>0$ Dersimonian-Laird random effects estimates (indicated as Summary RE) are presented; .p-values shown for results that are statistically significant with $\mathrm{p}<0.05$. 
are at higher likelihood of seropositivity to SARS-CoV-2 than of White HCWs. By studying HCWs across five hospitals with weekly symptom and serological assessment using two assays, case ascertainment was robust and the risk of either symptom recall bias or false positive or negative serological testing was minimised. Investigating a hospital HCW cohort with broad representation across different employment roles partially corrects for differences in exposure, socio-economic status, use of protective equipment and body mass index between ethnic groups, which was further addressed by adjusting for IMD.

Multiple previous studies have highlighted higher rates of hospitalisation for SARS-COV-2 infection across different BAME groups than would be expected from local demographics [1,23], with worse outcomes in hospitalised COVID-19 patients [2]. More recently epidemiological studies using primary care data (the largest including data from over 17.5 million people across the UK) have shown higher rates of test positivity for SARS-COV-2 in Black [24,5], as well as Asian and other ethnic minority groupings. All of the studies using retrospective analysis of routinely-collected healthcare record data are however subject to bias from ethnic differences in access to testing and healthcare alongside co-morbidities, reducing the accuracy of ascertainment of 'true' rates of infection. Better assessments of population infection rates can be obtained from large-scale prospective epidemiological studies with surveillance serology testing, with UK data from the REACT-2 study [4] using lateral flow immunoassay IgG to SARSCOV-2 finding point seroprevalence rates of $17.3 \%$ in people of Black ethnicity, although differentiating biological from socio-economic explanations behind these differences is challenging [25,26,27].

Our data, which have not explored differences in severity but only in seropositivity, do not exclude that socio-economic factors and in particular, health inequalities and higher rates of cardiometabolic or pulmonary comorbidities lead to the increased incidence and severity of SARS-CoV-2 infection (hospitalisations and mortality) observed in certain ethnic minority groups [28].

A potential study limitation was that the prevalence of specific comorbidities which was not included in the analysis. This was due to the different way these were collected in the two cohorts making a meaningful meta-analysis difficult to carry out. However, in each individual cohort no significant association between seroprevalence over the 10-13 weeks of observation and individual comorbidities was observed. Moreover, we find no association with BMI which is strongly correlated with most cardiometabolic and respiratory comorbidities (Table 1), therefore this is unlikely to have biased our results.

The reasons for increased rates of infection among Black HCWs may represent a combination of behavioural and biological factors. However, the lack of independent association with socioeconomic and other surrogates of behavioural confounding suggests a role for biological factors. This in turn suggests ethnic/heritable differences in resistance or protective immunity that may ultimately provide direct correlates of adaptive response to infection or vaccine mediated protection. Differences in innate immunity between individuals of European and African descent have been described and the functional genetic variations identified [29]. Moreover, differences in adaptive immune response have also been reported [30] specifically in the context of response to vaccination. Such data combined with ours suggests that individuals from some ethnic groups may be more likely to mount an antibody response to an asymptomatic or paucisymptomatic infection where individuals with a different genetic or ethnic background may use an innate immune response at the same level of exposure.

Future studies regarding the duration of neutralizing antibodies in different ethnic groups are necessary. The study failed to find differences in seropositivity across other ethnic minority groups, and point to the fact that the different rates of severe disease and mortality observed among Asians cannot be explained by increased rates of infection. It is likely that, independent of risk of infection, comorbid conditions and socio-economic factors likely play the main role in the higher susceptibility to a more severe form of Covid-19 among Asians [31]. This highlights the importance of disaggregating broad ethnic groupings such as BAME to better understand [6,32 root causes behind ethnic differences [24].

We further confirm the clear protective effect of working in ITU compared with other roles [6] during the first wave of the pandemic, with no differences seen between other occupational roles (doctors, nurses and allied healthcare professionals or clinical support staff), including front-line roles with responsibility for patients with COVID-19. This provides confidence in the validity of our data and suggests that the early prioritisation of higher grades of PPE to ITU staff [33], alongside differences in behaviours (social distancing and hand hygiene) [34,35] offered effective protection against nosocomial infections, and that such measures on subsequent waves could achieve similar protection for all staff.

There are several strengths of the current study. The seroprevalence data reflects five hospitals in two regions of the UK, and covers seropositivity over a period of 3 months during the first wave of the pandemic. The cohort had high exposure to SARS-CoV-2 and low incidence of co-morbidities. The study was prospective with weekly serial testing enabling understanding of infection timing and providing confidence in accuracy of overall infection and seroconversion rates over the period of study (rather than point prevalence). We have been able to adjust for confounders such as socioeconomic status and hospital role, location, (which one of the key determinants of differences in severity of Covid-19) [36] and contemporaneous collection of symptom data (rather than retrospective collection used in other studies) [32] improved confidence in symptom correlation with infection and reduced recall bias. We also note some study limitations. The data reported here focuses on mild (pauci-symptomatic) and asymptomatic infections and does not touch on the important issue of higher mortality and severity observed among HCWs belonging to ethnic minorities during the pandemic. This however has enabled us to focus on transmission at what is expected to be a relatively homogenous exposure level, and minimises the impact of health behaviours or access to care. Moreover, we have due to sample size constraints been unable to investigate links between atypical symptoms and seropositivity. Across both cohorts, classification of ethnic minority grouping was self-reported. Categories were broad this may hide potential biological impacts (for example between African versus Caribbean participants), however larger studies would be needed to address this comprehensively. The current study included 59 participants of self-reported Black ethnicity and therefore further studies on larger sample sizes will also be required to confirm generalisability of the current findings to the general population.

Rates of seroconversion to SARS-CoV-2 are higher in healthcare workers of Black ethnicity compared with their White colleagues, even after adjustment for age, sex, socio-economic status and occupational role. This difference was not present for HCWs from other ethnic minority groups. These results support biological explanations for differences in asymptomatic and paucisymptomatic response to SARS-CoV-2 infection between different ethnic groups, and warrant further study, particularly in light of vaccine-mediated protection.

\section{Authors' contributions}

Obtained funding: AMV, BJO, CMa, JCM

Conceived and designed the experiments: AMV, JCM, TAT CMa

Collected and processed samples: AN, AI, SC, JN, LC, PT, TAT, JCM,

Analysed the data: AMV, CMa, AN

Contributed reagents/materials/analysis tools: TDS, JW, CS, AM, TB, AS

Wrote the manuscript: AMV, JCM, CMa 
Revised the manuscript: All. AMV and CMa are responsible for the overall content as guarantors. The guarantors accept full responsibility for the work and/or the conduct of the study, had access to the data, and controlled the decision to publish. The manuscript's guarantors affirm that the manuscript is an honest, accurate, and transparent account of the study being reported; that no important aspects of the study have been omitted; and that any discrepancies from the study as planned have been explained.

The corresponding author attests that all listed authors meet authorship criteria and that no others meeting the criteria have been omitted.

\section{Funding}

Funding for the PANTHER study was from the UKRI/MRC (Cov0331 - MR/V027883/1), with additional institutional support from the Nottingham NIHR BRC. Funding for COVIDsortium was donated by individuals, charitable Trusts, and corporations including Goldman Sachs, Citadel and Citadel Securities, The Guy Foundation, GW Pharmaceuticals, Kusuma Trust, and Jagclif Charitable Trust, and enabled by Barts Charity with support from UCLH Charity. Wider support is acknowledged on the COVIDsortium website. Serology tests (anti-S1 and anti-NP) were funded by Public Health England. JCM, CMa and TAT are directly and indirectly supported by the University College London Hospitals (UCLH) and Barts NIHR Biomedical Research Centres and through the British Heart Foundation (BHF) Accelerator Award (AA/18/6/34223). TAT is funded by a BHF Intermediate Research Fellowship (FS/19/35/34374). MN is supported by the Wellcome Trust (207511/Z/17/Z) and by NIHR Biomedical Research Funding to UCL and UCLH. C Menni is supported by the Chronic Disease Research Foundation (CDRF).

\section{Data sharing statement}

Applications for access to the individual participant de-identified data (including data dictionaries) and samples from COVIDsortium can be made to the access committee via an online application https://covid-consortium.com/application-for-samples/. Each application will be reviewed, with decisions to approve or reject an application for access made on the basis of (i) accordance with participant consent and alignment to the study objectives (ii) evidence for the capability of the applicant to undertake the specified research and (iii) availability of the requested samples. The use of all samples and data will be limited to the approved application for access and stipulated in the material and data transfer agreements between participating sites and investigators requesting access.

\section{Declaration of competing interests}

Dr. Norrish reports grants from Medical Research Council, COVID19 Rapid Response Rolling Call, during the conduct of the study; personal fees from AOTrauma, personal fees from LINK Orthopaedics, outside the submitted work. Dr. Chaturvedi reports grants from Medical Research Council, grants from UKRI, personal fees from AstraZeneca, outside the submitted work. All other authors have nothing to declare.

\section{Supplementary materials}

Supplementary material associated with this article can be found, in the online version, at doi:10.1016/j.eclinm.2021.100835.

\section{References}

[1] Price-Haywood EG, Burton J, Fort D, Seoane L. Hospitalization and Mortality among Black Patients and White Patients with Covid-19. N Engl J Med 2020;382:2534-43.
[2] Harrison EM, Docherty AB, Barr B, et al. Ethnicity and Outcomes from COVID-19: The ISARIC CCP-UK Prospective Observational Cohort Study of Hospitalised Patients. 2020; published online May 31. DOI:10.2139/ssrn.3618215.

[3] Rimmer A. Covid-19: Disproportionate impact on ethnic minority healthcare workers will be explored by government. BMJ 2020;369:m1562.

[4] Ward H, Atchison CJ, Whitaker M, et al. Antibody prevalence for SARS-CoV-2 in England following first peak of the pandemic: REACT2 study in 100,000 adults. Infectious Diseases (except HIV/AIDS) 2020 published online Aug 14. doi: 10.37473/dac/10.1101/2020.08.12.20173690.

[5] de Lusignan S, Dorward J, Correa A, et al. Risk factors for SARS-CoV-2 among patients in the Oxford Royal College of General Practitioners Research and Surveillance Centre primary care network: a cross-sectional study. Lancet Infect Dis 2020:20:1034-42.

[6] Shields A, Faustini SE, Perez-Toledo M, et al. SARS-CoV-2 seroprevalence and asymptomatic viral carriage in healthcare workers: a cross-sectional study. Thorax 2020 published online Sept 11. doi: 10.1136/thoraxjnl-2020-215414.

[7] Lackermair K, William F, Grzanna N, et al. Infection with SARS-CoV-2 in primary care health care workers assessed by antibody testing. Fam Pract 2020 published online Aug 7 10.1093/fampra/cmaa078.

[8] Nguyen LH, Drew DA, Graham MS, et al. Risk of COVID-19 among front-line health-care workers and the general community: a prospective cohort study. Lancet Public Health 2020;5:e475-83.

[9] Khalil A, Hill R, Ladhani S, Pattisson K, O’Brien P. COVID-19 screening of healthcare workers in a London maternity hospital. Lancet Infect Dis 2020 published online May 18. doi: 10.1016/S1473-3099(20)30403-5.

[10] Barrett ES, Horton DB, Roy J, et al. Prevalence of SARS-CoV-2 infection in previously undiagnosed health care workers at the onset of the U.S. COVID-19 epidemic. medRxiv 2020 published online April 24. doi: 10.1101/ 2020.04.20.20072470.

[11] Toner E, Waldhorn R. [No title]. 2020; published online Feb 27. https://centerforhealthsecurity.org/cbn/2020/cbnreport-02272020.html (accessed Oct 29, 2020).

[12] Peate I. NHS workforce race equality. Br J Nurs 2020;29:81.

[13] Mahase E. Covid-19: Ethnic minority doctors feel more pressured and less protected than white colleagues, survey finds. BMJ 2020;369:m2506.

[14] Panther Study - Pandemic Tracking of Healthcare Workers. https://pantherstudy. org.uk/(accessed Oct 29, 2020).

[15] Augusto JB, Menacho K, Andiapen M, et al. Healthcare Workers Bioresource: Study outline and baseline characteristics of a prospective healthcare worker cohort to study immune protection and pathogenesis in COVID-19. Wellcome Open Research 2020;5:179.

[16] Beavis KG, Matushek SM, Abeleda APF, et al. Evaluation of the EUROIMMUN AntiSARS-CoV-2 ELISA Assay for detection of IgA and IgG antibodies. J Clin Virol 2020;129:104468.

[17] Ainsworth M, Andersson M, Auckland K, et al. Performance characteristics of five immunoassays for SARS-CoV-2: a head-to-head benchmark comparison. Lancet Infect Dis 2020;20:1390-400.

[18] Haselmann V, Kittel M, Gerhards C, et al. Comparison of test performance of commercial anti-SARS-CoV-2 immunoassays in serum and plasma samples. Clin Chim Acta 2020;510:73-8.

[19] Public Health England. COVID-19: laboratory evaluations of serological assays. 2020; published online May 19. https://www.gov.uk/government/publications/ covid-19-laboratory-evaluations-of-serological-assays. (accessed Dec 17, 2020).

[20] Menni C, Valdes AM, Freidin MB, et al. Real-time tracking of self-reported symptoms to predict potential COVID-19. Nat Med 2020;26:1037-40.

[21] McLennan D, Noble S, Noble M, Plunkett E, Wright G, Gutacker N. The English Indices of Deprivation 2019: technical report. 2019. https://dera.ioe.ac.uk/34259/ 1/IoD2019_Technical_Report.pdf.

[22] Gender in the NHS infographic. https://www.nhsemployers.org/case-studiesand-resources/2019/05/gender-in-the-nhs-infographic (accessed March 3, 2021).

[23] Karaca-Mandic P, Georgiou A, Sen S. Assessment of COVID-19 Hospitalizations by Race/Ethnicity in 12 States. JAMA Intern Med 2020 published online Aug 17. doi: 10.1001/jamainternmed.2020.3857.

[24] Mathur R, Rentsch CT, Morton C, et al. Ethnic differences in COVID-19 infection, hospitalisation, and mortality: an OpenSAFELY analysis of 17 million adults in England. medRxiv2020. DOI:10.1101/2020.09.22.20198754

[25] Perkin MR, Heap S, Crerar-Gilbert A, et al. Deaths in people from Black, Asian and minority ethnic communities from both COVID-19 and non-COVID causes in the first weeks of the pandemic in London: a hospital case note review. BMJ Open 2020;10:e040638.

[26] Baumer T, Phillips E, Dhadda A, Szakmany T. Epidemiology of the First Wave of COVID-19 ICU Admissions in South Wales-The Interplay Between Ethnicity and Deprivation. Front Med 2020;7:569714.

[27] Otu A, Ahinkorah BO, Ameyaw EK, Seidu A-A, Yaya S. One country, two crises: what Covid-19 reveals about health inequalities among BAME communities in the United Kingdom and the sustainability of its health system? Int J Equity Health 2020;19:189.

[28] Weekly national Influenza and COVID19 surveillance report: week 51. https:// assets.publishing.service.gov.uk/government/uploads/system/uploads/attachment_data/file/945502/Weekly_Flu_and_COVID-19_report_w51_FINAL.pdf.

[29] Quach H, Rotival M, Pothlichet J. Genetic Adaptation and Neandertal Admixture Shaped the Immune System of Human Populations. Cell 2016;167:643-56 e17.

[30] Happe M, Samuvel DJ, Ohtola JA, Korte JE, Westerink MAJ. Race-related differences in functional antibody response to pneumococcal vaccination in HIV-infected individuals. Vaccine 2019;37:1622-9.

[31] Lassale C, Gaye B, Hamer M, Gale CR, Batty GD. Ethnic disparities in hospitalisation for COVID-19 in England: The role of socioeconomic factors, mental health, 
A.M. Valdes et al. / EClinicalMedicine 00 (2021) 100835

and inflammatory and pro-inflammatory factors in a community-based cohort study. Brain Behav Immun 2020;88:44-9.

[32] Ward H, Atchison CJ, Whitaker M, et al. Antibody prevalence for SARS-CoV-2 in England following first peak of the pandemic: REACT2 study in 100,000 adults. medRxiv 2020 2020.08.12.20173690.

[33] Cook TM. Personal protective equipment during the coronavirus disease (COVID) 2019 pandemic - a narrative review. Anaesthesia 2020;75:920-7.

[34] Bansal P, Bingemann TA, Greenhawt M. Clinician Wellness During the COVID-19 Pandemic: Extraordinary Times and Unusual Challenges for the Allergist/Immunologist. J Allergy Clin Immunol Pract 2020;8:1781-90 e3.
[35] Morawska L, Tang JW, Bahnfleth W, et al. How can airborne transmission of COVID-19 indoors be minimised? Environ Int 2020;142:105832.

[36] Ayoubkhani D, Nafilyan V, White C, et al. Ethnic minority groups in England and Wales - factors affecting the size and timing of elevated COVID-19 mortality: a retrospective cohort study linking Census and death records. bioRxiv 2020 published online Aug 4.. doi: 10.1101/2020.08.03.20167122. 